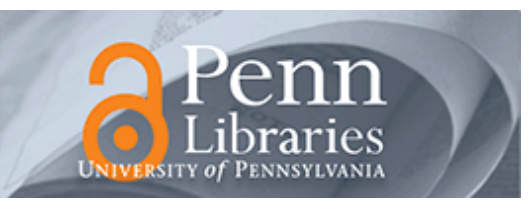

University of Pennsylvania

ScholarlyCommons

$11-1-2010$

\title{
Use of a Single Multiplexed CMOS Oscillator as Direct Frequency Read-Out for an Array of Eight AIN Contour-Mode NEMS Resonant Sensors
}

\author{
Matteo Rinaldi \\ University of Pennsylvania, rinaldim@seas.upenn.edu \\ Chiara Zuniga \\ University of Pennsylvania, zunigac@seas.upenn.edu \\ Brandon Duick \\ University of Pennsylvania, duick@seas.upenn.edu \\ Gianluca Piazza \\ University of Pennsylvania, piazza@seas.upenn.edu \\ Follow this and additional works at: https://repository.upenn.edu/ese_papers \\ Part of the Engineering Science and Materials Commons
}

\section{Recommended Citation}

Matteo Rinaldi, Chiara Zuniga, Brandon Duick, and Gianluca Piazza, "Use of a Single Multiplexed CMOS Oscillator as Direct Frequency Read-Out for an Array of Eight AIN Contour-Mode NEMS Resonant Sensors", . November 2010.

\section{Suggested Citation:}

Rinaldi, Matteo; Zuniga, Chiara; Duick, Brandon; Piazza, Gianluca; , "Use of a single multiplexed CMOS oscillator as direct frequency read-out for an array of eight AIN Contour-Mode NEMS Resonant Sensors," Sensors, 2010 IEEE, vol., no., pp.2666-2670, 1-4 Nov. 2010.

C2010 IEEE. Personal use of this material is permitted. However, permission to reprint/republish this material for advertising or promotional purposes or for creating new collective works for resale or redistribution to servers or lists, or to reuse any copyrighted component of this work in other works must be obtained from the IEEE.

This paper is posted at ScholarlyCommons. https://repository.upenn.edu/ese_papers/591

For more information, please contact repository@pobox.upenn.edu. 


\title{
Use of a Single Multiplexed CMOS Oscillator as Direct Frequency Read-Out for an Array of Eight AIN Contour-Mode NEMS Resonant Sensors
}

\author{
Abstract \\ This paper reports on the first demonstration of a single multiplexed CMOS oscillator circuit employed as \\ direct frequency readout for an array of 8 nanoscaled aluminum nitride Contour-Mode Resonant Sensors \\ (CMR-S). In this first prototype 8 thin-film $(250 \mathrm{~nm}$ ) AIN CMR-S operating at $186 \mathrm{MHz}$ were fabricated on \\ the same chip and simultaneously wire-bonded to a Pierce-like oscillator circuit (fabricated in the ON \\ Semiconductor $0.5 \mu \mathrm{m}$ CMOS process) by means of 8 CMOS transmission gates addressed via a 3 bit on- \\ chip decoder. The $8 \mathrm{CMR}-\mathrm{S}$ were simultaneously exposed to different concentrations of methanol \\ $(0.1-1 \%$ of the saturated vapor pressure) and their response was monitored in a time-multiplexed mode. \\ Frequency shifts of $300 \mathrm{~Hz}$ corresponding to changes of mass per unit area of $7 \mathrm{ag} / \mathrm{\mu m}^{2}$ were \\ experimentally detected. Values of phase noise derived Allan deviation as low as $0.9 \mathrm{~Hz}$ were measured. \\ Such Allan deviation translates in an estimated limit of detection of $21 \mathrm{zg} / \mathrm{\mu m}^{2}$.

\section{Disciplines} \\ Engineering | Engineering Science and Materials

\section{Comments} \\ Suggested Citation: \\ Rinaldi, Matteo; Zuniga, Chiara; Duick, Brandon; Piazza, Gianluca; , "Use of a single multiplexed CMOS \\ oscillator as direct frequency read-out for an array of eight AIN Contour-Mode NEMS Resonant Sensors," \\ Sensors, 2010 IEEE , vol., no., pp.2666-2670, 1-4 Nov. 2010. \\ (C2010 IEEE. Personal use of this material is permitted. However, permission to reprint/republish this \\ material for advertising or promotional purposes or for creating new collective works for resale or \\ redistribution to servers or lists, or to reuse any copyrighted component of this work in other works must \\ be obtained from the IEEE.
}




\title{
Use of a Single Multiplexed CMOS Oscillator as Direct Frequency Read-Out for an Array of Eight AIN Contour-Mode NEMS Resonant Sensors
}

\author{
Matteo Rinaldi, Chiara Zuniga, Brandon Duick and Gianluca Piazza \\ Department of Electrical and Systems Engineering \\ University of Pennsylvania \\ Philadelphia, USA \\ rinaldim@seas.upenn.edu
}

\begin{abstract}
This paper reports on the first demonstration of a single multiplexed CMOS oscillator circuit employed as direct frequency readout for an array of 8 nanoscaled aluminum nitride Contour-Mode Resonant Sensors (CMR-S). In this first prototype 8 thin-film $(250 \mathrm{~nm})$ AIN CMR-S operating at 186 MHz were fabricated on the same chip and simultaneously wirebonded to a Pierce-like oscillator circuit (fabricated in the ON Semiconductor $0.5 \mu \mathrm{m}$ CMOS process) by means of $8 \mathrm{CMOS}$ transmission gates addressed via a 3 bit on-chip decoder. The 8 CMR-S were simultaneously exposed to different concentrations of methanol (0.1-1\% of the saturated vapor pressure) and their response was monitored in a time-multiplexed mode. Frequency shifts of $300 \mathrm{~Hz}$ corresponding to changes of mass per unit area of $7 \mathrm{ag} / \mu \mathrm{m}^{2}$ were experimentally detected. Values of phase noise derived Allan deviation as low as $0.9 \mathrm{~Hz}$ were measured. Such Allan deviation translates in an estimated limit of detection of $21 \mathrm{zg} / \mu \mathrm{m}^{2}$.
\end{abstract}

\section{INTRODUCTION}

In recent years the demand for smart, compact, portable and low cost electronic noses for multiple gas detection has steadily grown. Sensory systems that monitor frequency shifts, such as Quartz Crystal Microbalances (QCMs), have a significant advantage over other sensor technologies (conductance-based sensors, Chem-FET, or optical sensors) since they use frequency as the output variable, which is one of the physical quantities that can be monitored with the highest accuracy [1]. Nevertheless, smart, compact and portable electronic noses cannot be implemented with bulky and unintegrable QCMs and require the employment of highly miniaturized sensor arrays capable of detecting extremely small concentrations (part per trillion, ppt) of gaseous analytes.

NanoElectroMechanical Systems (NEMS) resonators [2, 3] have been exploited as transducers for the realization of extremely sensitive miniaturized gravimetric sensors. Nevertheless, the greatly reduced dimensions of these devices render their transduction extremely difficult and require the use of cumbersome, complex and power inefficient read-out techniques. This limitation has prevented the fabrication of NEMS resonant sensor arrays with compact and power efficient read-out systems for simultaneous monitoring of all the sensing elements.

In this perspective, the employment of large surface area devices with nanoscaled thickness (such as nano-CMR-S) [4, 5], instead of beams, is advantageous since it enables the fabrication of extremely low mass devices with power efficient transduction and read-out.

In this work direct frequency read-out of an array of NEMS resonant sensors is demonstrated for the first time by simultaneously connecting 8 nanoscaled AIN CMR-S to a single Pierce-like CMOS oscillator operating in a timemultiplexed mode (Fig. 1).

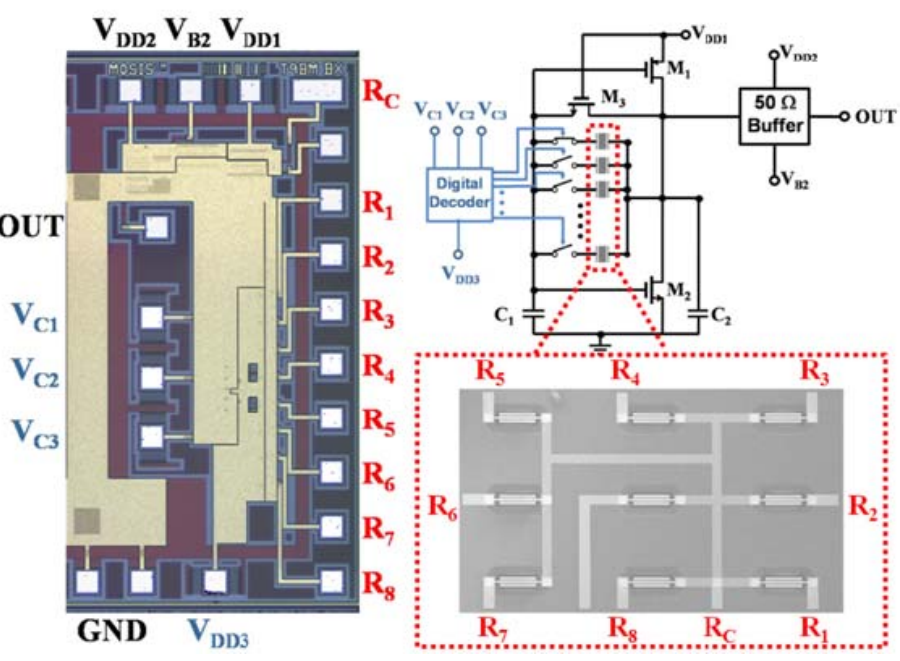

Figure 1. Micrograph and schematic circuit representation of the multiplexed CMOS oscillator chip $\left(1.05 \mathrm{~mm}^{2}\right)$. The zoomed-in view shows the SEM picture of the nano-CMR-S array $\left(1.5 \mathrm{~mm}^{2}\right)$. 
The 8 nano-CMR-S, operating at $186 \mathrm{MHz}$, were simultaneously exposed to different concentrations of methanol and the response of each device was sequentially acquired with a sampling time as low as $100 \mathrm{~ms}$. Frequency shifts of $300 \mathrm{~Hz}$, corresponding to changes of mass per unit area of $\sim 7 \mathrm{ag} / \mu^{2}$, were experimentally detected. Values of phase noise derived Allan deviation as low as $0.9 \mathrm{~Hz}$ for $10 \mathrm{~ms}$ gate time were measured. This Allan deviation translates in an estimated limit of detection of only $21 \mathrm{zg} / \mathrm{\mu m}^{2}$. This CMR-S array prototype is approximately 4 orders of magnitude smaller in volume compared to a previously demonstrated electronic nose based on multichannel QCMs [6].

\section{DESIGN}

\section{A. Nano-CMR-S Array}

A CMR is composed of an AIN film sandwiched between two patterned metal electrodes (Fig. 2). When an AC votlage is applied across the thickness $(T)$ of the device a contour-extensional mode of vibration is excited through the equivalent $d_{31}$ piezoelectric coefficient of AlN.

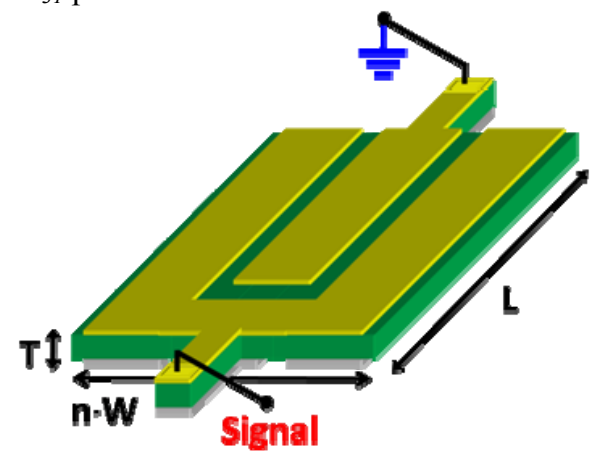

Figure 2. Schematic representation of a $186 \mathrm{MHz}$ nano-CMR-S composing the array: $T=250 \mathrm{~nm}, W=20 \mu \mathrm{m}, L=200 \mu \mathrm{m}, n=3$.

Given the equivalent mass density, $\rho_{e q}$, and Young's modulus, $E_{\text {eq }}$, of the material stack (AlN and metal electrodes) that forms the resonator, the center frequency, $f_{0}$, of this laterally vibrating mechanical structure, is univocally set by the period, $W$, of the metal electrode patterned on the AIN plate and can be approximately expressed as in Eq. (1).

$$
f_{0}=\frac{1}{2 W} \sqrt{\frac{E_{e q}}{\rho_{e q}}}
$$

The other two geometrical dimensions, thickness, $T$, and length, $L$, set the equivalent electrical impedance of the resonator [7] and can be designed independently of the desired resonance frequency.

The sensitivity to mass per unit area of a CMR-S loaded on its top surface can be expressed as in Eq. (2) [8, 9].

$$
\begin{gathered}
S_{C M R-S}=-\frac{f_{0}}{2 \rho_{e q} T} \\
L O D=\frac{\Delta f_{\min }}{S_{C M R-S}}
\end{gathered}
$$

It has been shown in $[4,5]$ that when the resonant sensor is connected to a self-sustained oscillator loop for direct frequency readout, its performance, in terms of limit of detection, LOD (Eq. 3), can be improved by reducing the thickness, $T$, of the AlN layer up to the limit for which good phase noise is preserved (the minimum detectable frequency shift, $\Delta f_{\min }$, is in fact determined by the phase noise of the oscillator). According to this, the thicknesses, $T$, of the CMR$\mathrm{S}$ of this work were scaled into the nano domain $(250 \mathrm{~nm})$ and, despite the volume reduction, high $Q$ values $(\sim 1,000$ in air), which directly affect phase noise, were still attained. In addition, in order to maximize the transduction efficiency of the nanoscaled piezoelectric resonator a thickness field excitation scheme (TFE) [9] was employed to excite a higher order contour-extensional mode of vibration in the nanoscaled AlN structures. This allowed achieving values of the electromechanical coupling coefficient, $k_{t}^{2}$, as high as $2 \%$. Such high values $(\sim 20)$ of the device figure of merit, $k_{t}^{2} \cdot Q$, are of crucial importance for the direct connection of multiple CMR-S to a compact and low power multiplexed oscillator circuit for direct frequency read-out. In fact, the primary power loss in such oscillator circuit is due to the motional resistance, $R_{m}$, of the resonator [10], whose value is inversely proportional to the device figure of merit, $k_{t}^{2} \cdot Q[11,12]$.

\section{B. Multiplexed CMOS Oscillator}

The oscillator circuit topology used as read-out for the CMR-S array of this work is shown in Figure 1. The circuit consists of a Pierce oscillator implemented by means of a CMOS inverter biased in its active region. Transistors $M I$ and $M 2$ form the CMOS inverting amplifier while transistor $M 3$ acts as a large resistor to provide biasing of $M 1$ and $M 2$ in the active region. By employing this circuit topology the transconductance, $g_{m}$, of the inverting amplifier is made linearly proportional to the supply voltage $V_{D D 1}$ [10], which allows optimizing the oscillator performance in terms of power consumption and phase noise depending upon the characteristics of the specific NEMS resonator connected in the feedback loop. By adjusting $V_{D D 1}$ the $\mathrm{AC}$ gain of the inverting amplifier can be set to be equal or above the critical transconductance, $g_{m c}$, needed for the oscillations to start.

The eight nano AlN CMR-S are simultaneously connected to the Pierce-like oscillator circuit by means of an equivalent number of CMOS switches (Fig. 1) operating in a time multiplexed mode. Each switch is composed of a CMOS transmission gate whose dimensions are opportunely designed in order to minimize power loss and consequently maintain a low value of gain in the amplifier used to sustain the oscillation. In particular, by acting on the $W / L$ ratio of the transistors composing the transmission gates the values of onresistance and the input/output capacitance of the switches can be opportunely set in order to minimize power dissipation. A large $W / L$ ratio reduces the on-resistance of the switches (hence power loss), but at the same time increases the values of their input/output capacitance, $C_{p}$, which, as shown in Figure 3, needs instead to be kept much smaller than the resonator geometrical capacitance, $C_{0}$, in order to limit excessive power dissipation. Furthermore, all the switch 
input/output capacitances, $C_{p}$, add up in parallel contributing to the total value of capacitance to ground represented by the capacitors $C_{1}$ and $C_{2}$ in Figure 1.

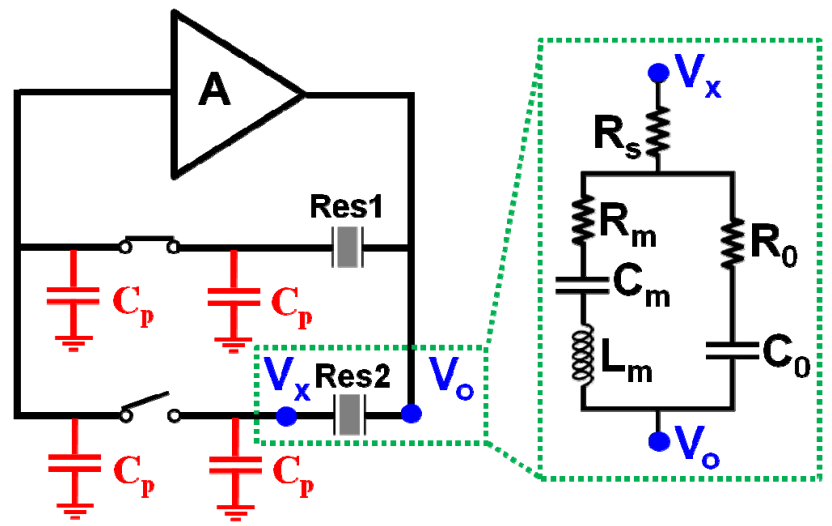

Figure 3. Schematic representation of two nano-CMR-S, Res 1 and Res2, connected to the CMOS inverting amplifier, $A$, by means of two CMOS switches. In order to have $V_{o} \approx V_{x}$ (i.e. no power dissipation in the turned off resonator) the resonator geometrical capacitance, $C_{0}$, has to be much larger than $C_{p}$. Although just 2 resonators are shown in this schematic, 8 were effectively connected in the prototype presented in this paper.

The capacitances $C_{1}$ and $C_{2}$ (assumed to be equal), are necessary to provide the adequate phase shift in the Pierce oscillator configuration $[13,14]$, and their value affects the critical transconducatance, $g_{m c}$, that the inverting amplifier needs to provide in order to sustain the oscillation. In fact, for a given value of the device capacitance, $C_{0}$, an optimum value of $C_{1}$ can be found in order to achieve stable oscillation with minimum $g_{m}$ (i.e. minimum power consumption) (Fig. 4).

The nanoscaled devices forming the array presented in this work are characterized by a geometrical capacitance, $C_{0}$, equal to approximately $3.4 \mathrm{pF}$ and a figure of merit, $k_{t}^{2} \cdot Q$, of 20 , which translates in a value of motional resistance, $R_{m}$, equal to16 $\Omega$ [12]. Therefore, the inverting amplifier needs to provide a negative resistance, $R_{E N}$, less than $-16 \Omega$ in order to sustain the oscillation [14]. In Figure 4 the negative resistance provided by the CMOS circuit is plotted as a function of the $C_{0} / C_{1}$ ratio. The optimum value of $C_{1}$ (for which stable oscillation with minimum $g_{m}$ is achieved) is found to be approximately $C_{0} / 2.5$. The value of $C_{1}$ and $C_{2}$ depends on the parallel combination of different contributions coming from the gate-source and drain-source capacitances of transistors $M 1$ and $M 2$, input-to-output (junction) capacitances of all the CMOS switches connected in the feedback loop, interconnects capacitances, bonding pads capacitances, parasitic capacitances due to wire-bonding and any additional integrated or off-chip capacitance. Among all these contributions, the parasitic capacitance due to wire-bonding is a parameter that cannot be precisely controlled. Therefore, for an accurate and robust design of the oscillator (i.e. selecting the optimum value of $C_{1}$ for a given resonator $C_{0}$ ), it is necessary to make the other capacitive and well controlled contributions, such as the switch input/output capacitances or additional integrated or off-chip capacitances, dominant over the wire-bonding parasitic capacitance. In this work the parallel combination of the input/output capacitances, $C_{p}$, of the $8 \mathrm{CMOS}$ switches is used to set the value of $C_{1}$ and $C_{2}$ without the need of additional integrated or off-chip capacitors.

According to these considerations and given a minimum channel length, $L$, equal to $0.6 \mu \mathrm{m}$, set by the available CMOS technology, an optimum value for the width, $W$, of the transistors forming the switches was estimated to be approximately $56 \mu \mathrm{m}$ by means of circuit simulations performed in Cadence. This design choice corresponds to a value of $C_{p}$ of about $120 \mathrm{fF}$, which, given an estimated value of wire-bonding parasitic capacitance comprised between 300 and $700 \mathrm{fF}$, guarantees a value of $C_{0} / C_{1}$ ratio close to the optimum (hence the achievement of stable oscillations with minimum power consumption) without the need of additional integrated or off-chip capacitors.

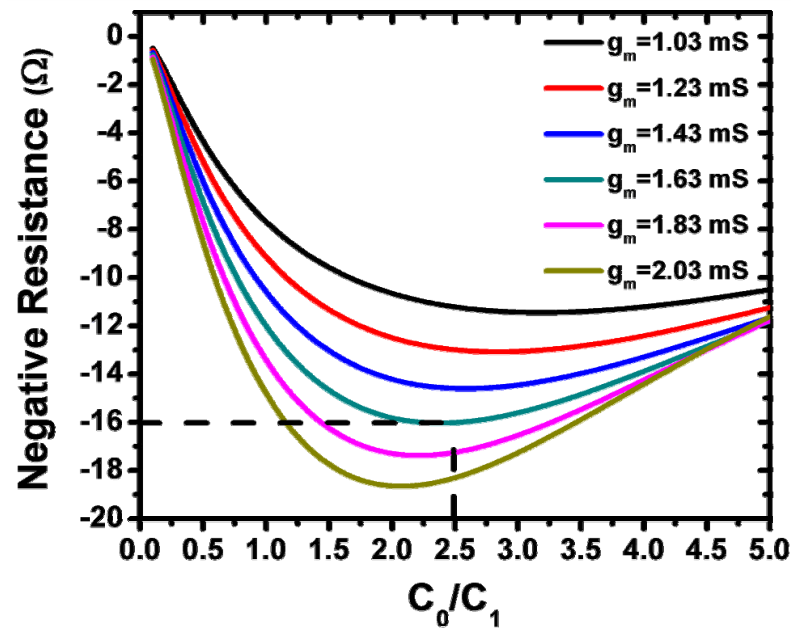

Figure 4. Negative resistance provided by the CMOS inverting amplifier plotted versus the $C_{0} / C_{1}$ ratio for different values of transconductance, $g_{m}$. The minimum value of $g_{m}$ which provides enough negative resistance to compensate the loss in the resonant device (i.e. $16 \Omega$ ) is found in correspondence of $\mathrm{C}_{0} / \mathrm{C}_{1} \approx 2.5$. Also note that this minimum is rather flat and slight variations in $C_{l}$ (such as changes in wire-bond capacitance) can be accommodated.

In order to reduce the number of pads necessary to control the sensor array, the CMOS switches are addressed trough a 3 to 8 digital decoder integrated on-chip (Fig. 1). Each nano$\mathrm{CMR}-\mathrm{S}$ is driven by the oscillator when the corresponding 3 bit address is presented to the decoder.

\section{EXPERIMENTAL RESULTS}

The eight nano AlN CMR-S operating at $186 \mathrm{MHz}$ were fabricated on a single chip with a process analogous to the one reported in [9]. The multiplexed CMOS oscillator chip was taped-out in the ON Semiconductor $0.5 \mu \mathrm{m}$ CMOS process. In order to test the sensing properties of the fabricated array of nano-CMR-S, the top metal electrode of each sensing element was made out of gold (50 nm thick), given its known ability to adsorb organic molecules in vapor phase [15]. Both the NEMS resonant sensor array die and the CMOS chip were attached to a custom designed PCB and all the electrical connections were made through wire-bonding.

The 8 combinations of the 3 bit address (corresponding to each of the nano-CMR-S in the array) were cyclically 
provided to the decoder by a Data Acquisition (DAQ) system so as to sequentially turn on each resonator. The output of the multiplexed oscillator was first monitored via an Agilent E5052B Signal Source Analyzer. The 8 resonators have similar center frequencies around $186 \mathrm{MHz}$ and the oscillator carrier frequency exhibits a standard deviation of $220 \mathrm{KHz}$ for the 8 nano-CMR-S. Also, unoptimized phase noise values for a single biasing voltage show variations between -96 and -70 $\mathrm{dBc} / \mathrm{Hz}$ at $1 \mathrm{KHz}$ offset and between -158 and $-150 \mathrm{dBc} / \mathrm{Hz}$ for offset greater than $300 \mathrm{KHz}$ (Fig. 5).

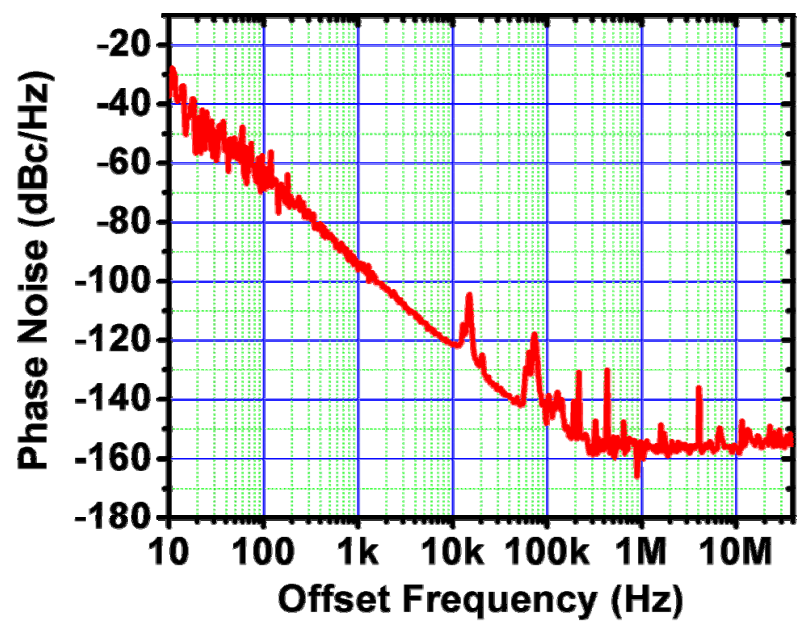

Figure 5. Typical phase noise response of one of the resonators connected to the CMOS multiplexed oscillator. Typical output power is around $-11 \mathrm{dBm}$.

The 8 nano-CMR-S were simultaneously exposed to methanol vapor (generated by bubbling technique) using the PCB itself as the lid for the testing chamber [9]. The response of each device was acquired in a time-multiplexed mode: the 8 combinations of 3 bit address (corresponding to each of the nano-CMR-S in the array) were cyclically (100 ms period) provided to the decoder by the DAQ and the frequency measurement was serially performed by an Agilent 53132A frequency counter connected to a computer (Fig. 6).

The nano-CMR-S array was exposed to different concentrations of methanol and a minimum frequency shift of approximately $300 \mathrm{~Hz}$ was detected for a $0.1 \%$ concentration of the methanol saturated vapor pressure (Fig. 7). Given each device has an average sensitivity around $42 \mathrm{KHz} \cdot \mathrm{\mu m}^{2} / \mathrm{fg}$ (extracted according to [9]) a limit of detection (LOD) of mass per unit area of $7 \mathrm{ag} / \mu^{2}$ was experimentally attained.

In order to estimate the noise-limited minimum frequency shift detectable by the sensor read-out $\left(\Delta f_{\min }\right)$, the short term frequency stability of the oscillator output signal was estimated by monitoring its phase-noise derived Allan deviation, $\sigma_{y}(\tau)$. An equivalent value of the Allan deviation as low as $\sim 0.9 \mathrm{~Hz}$ was obtained for a measurement time of 10 ms. Therefore, considering the device mass sensitivity of $\sim 42 \mathrm{KHz} \cdot \mu \mathrm{m}^{2} / \mathrm{fg}$ and a minimum detectable frequency shift of $0.9 \mathrm{~Hz}$, a LOD of mass per unit area of approximately $21 \mathrm{zg} / \mu^{2}$ can be estimated for the nano-CMR$\mathrm{S}$ composing the array presented in this work (Fig. 8).

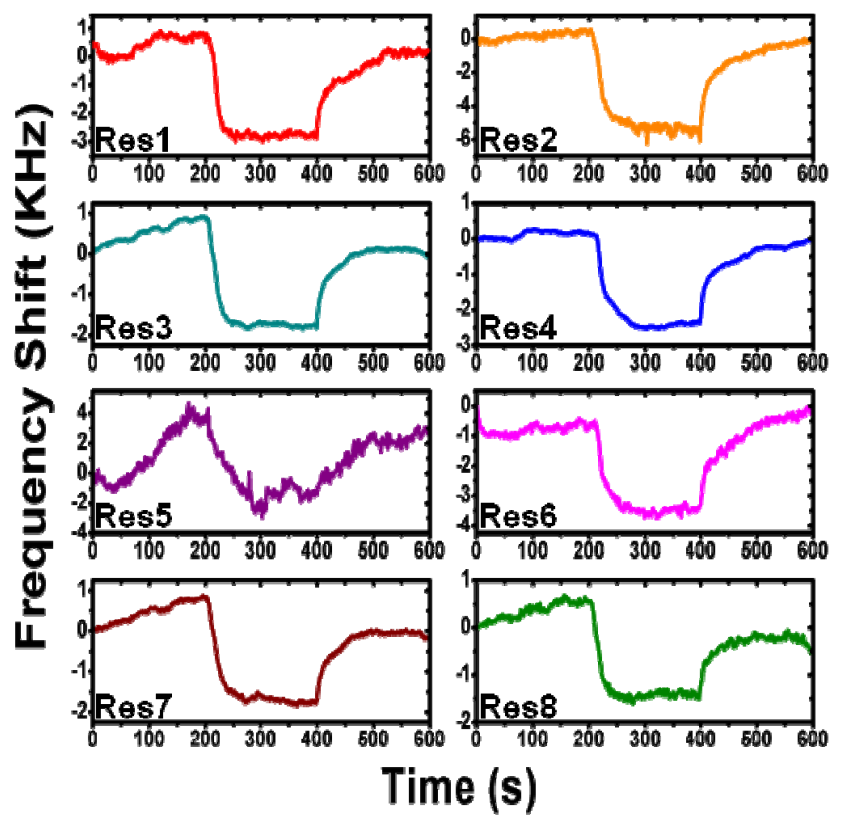

Figure 6. Measured response of the nano-CMR-S array exposed to $1 \%$ saturated vapor pressure of methanol andadsorbed on the top gold electrode of the devices. All 8 devices show similar responses. Different values of $V_{D D l}$ were employed during the testing in order to optimize the phase noise performances of different devices.

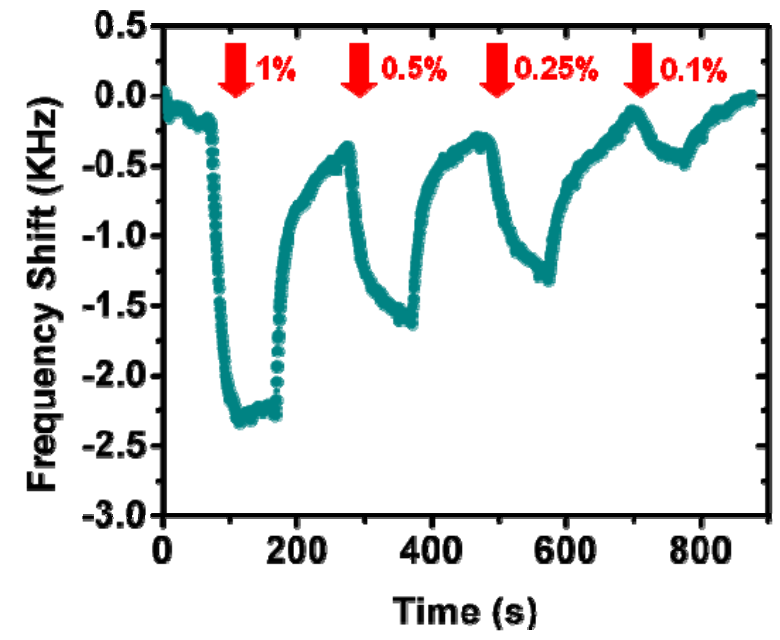

Figure 7. Measured response of one of the nano-CMR-S composing the array exposed to different concentrations (1-0.1\% saturated vapor pressure) of methanol. A minimum frequency shift of $\sim 300 \mathrm{~Hz}$ is detected in correspondence of the lowest concentration of methanol.

\section{CONCLUSION}

In this paper, the design and the experimental verification of the first multiplexed CMOS oscillator circuit employed as direct frequency readout for an array of 8 nanoscaled aluminum nitride Contour-Mode Resonant Sensors (CMR-S) was demonstrated. Design issues for the optimum implementation of such system were introduced and discussed. Thanks to the large value of the NEMS resonator figure of merit, $k_{t}^{2} \cdot Q \approx 20$, (despite the volume reduction) 
direct wire-bonding of 8 nano-CMR-S to a single multiplexed Pierce oscillator fabricated in the ON Semiconductor $0.5 \mu \mathrm{m}$ CMOS process was made possible. Phase noise values as low as $-96 \mathrm{dBc} / \mathrm{Hz}$ at $1 \mathrm{KHz}$ offset frequency and $-158 \mathrm{dBc} / \mathrm{Hz}$ for an offset frequency greater than $300 \mathrm{KHz}$ were measured. The 8 nano-CMR-S were simultaneously exposed to methanol vapor and the response of each device was acquired in a timemultiplexed mode showing the capability to detect values of adsorbed mass per unit area as low as $7 \mathrm{ag} / \mu \mathrm{m}^{2}$. Thanks to the high frequency stability of the oscillator signal (minimum Allan deviation of $0.9 \mathrm{~Hz}$ for $10 \mathrm{~ms}$ gate time), a LOD of mass per unit area of approximately $21 \mathrm{zg} / \mu^{2}$ was estimated for the nano-CMR-Ss composing the array presented in this work.

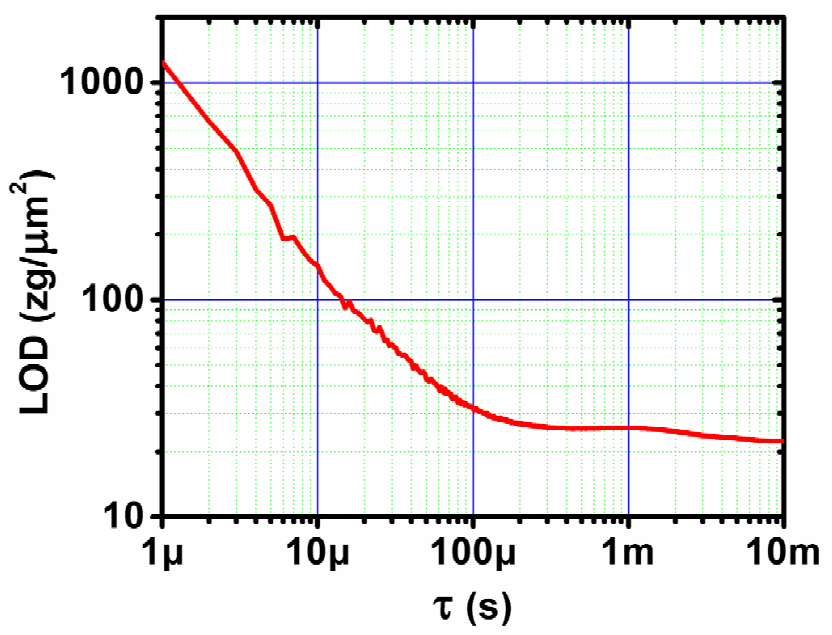

Figure 8. Limit of detection (LOD) for different values of integration time, $\tau$ (measurement speed), calculated from the measured phase noise (using an Agilent ${ }^{\circledR}$ E5052B Signal Source Analyzer) of one of the nano-CMR-S composing the array.

\section{ACKNOWLEDGMENT}

The authors thank Professor Jan Van der Spiegel, Chengjie Zuo and Xiaotie $\mathrm{Wu}$ for the helpful discussions on the multiplexed oscillator circuit design. We also thank the MOSIS Educational Program for the IC chip fabrication. The AlN deposition has been performed by Tegal Corporation.

\section{REFERENCES}

[1] J. R. Vig, L. Filler, Y. Kim, J. Microelectromech. Syst., vol. 5, no. 2, pp. 131-137, 1996.

[2] M. Li, H. X. Tang, M.L. Roukes, Nature Nanotechnology, vol. 2, pp. 114-120, 2007.

[3] P.S.Waggoner, H.G.Craighead, Lab on a Chip, vol. 7, p. 1238-1255, 2007.

[4] M. Rinaldi, B. Duick, C. Zuniga, C. Zuo and G. Piazza, Proc. $23^{\text {rd }}$ IEEE International Conference on Micro Electro Mechanical Systems (MEMS 2010), Jan. 2010, pp. 132-135.

[5] M. Rinaldi, C. Zuniga, C. Zuo and G. Piazza, Proc. Solid-State Sensors, Actuators, and Microsystems Workshop (Hilton Head 2010), Jun. 2010, pp. 471-474.

[6] E. Zampetti, S. Pantalei, A. Macagnano,C. Proietti, C. Di Natale, A. D’Amico, Sens . and Act. B,131, p.159-166, 2008.

[7] G. Piazza, P.J. Stephanou, A.P. Pisano, J. Microelectromech. Syst., vol. 15, no.6, pp. 1406-1418, 2006.

[8] C. Zuniga, M. Rinaldi, S. M. Khamis, A. T. Johnson, and G. Piazza, Applied Physics Letters, vol. 94, 223122, 2009.

[9] M. Rinaldi, C. Zuniga, and G. Piazza, Poc. IEEE International Ultrasonics Symposium 2009, Sept. 2009, pp. 714-717.

[10] C. Zuo, J. Van der Spiegel, and G. Piazza. IEEE Transactions on Ultrasonics, Ferroelectrics, and Frequency Control, vol. 57, no. 1, Jan. 2010, pp. 82-87.

[11] K.M. Lakin, Proc. IEEE MTT-S Int. Microwave Symp. Dig., June 1992, pp. 149-152.

[12] M. Rinaldi, C. Zuniga, C. Zuo and G. Piazza, IEEE Transactions on Ultrasonics, Ferroelectrics, and Frequency Control, vol. 57, no. 1, Jan. 2010, pp. 38-45.

[13] W. M. C. Sansen, Analog Design Essentials, New York: SpringerVerlag, 2006.

[14] E. A. Vittoz, M. G. R. Degrauwe and S. Bitz, IEEE J. Solid-State Circuits, vol. 23, no. 3, June 1988, pp. 774-783.

[15] T. M. Herne and M. J. Tarlov, J. Am. Chem. Soc., 119, pp. 8916-8920, 1997. 